\title{
Inhalt / Contents 01/03
}

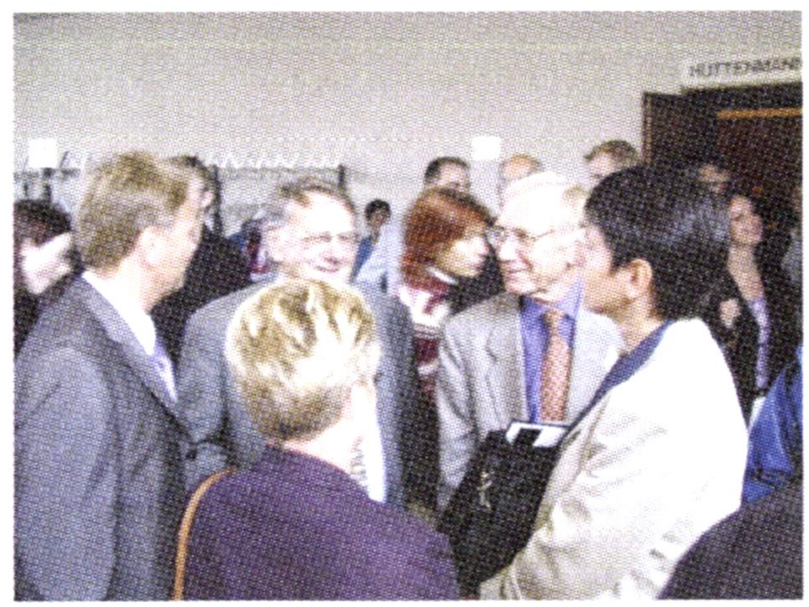

\section{S. Mücklich}

11. Internationale Metallographietagung in Leoben - Bildbericht

$11^{\text {th }}$ International Congress on Metallography at Leoben - A photo report

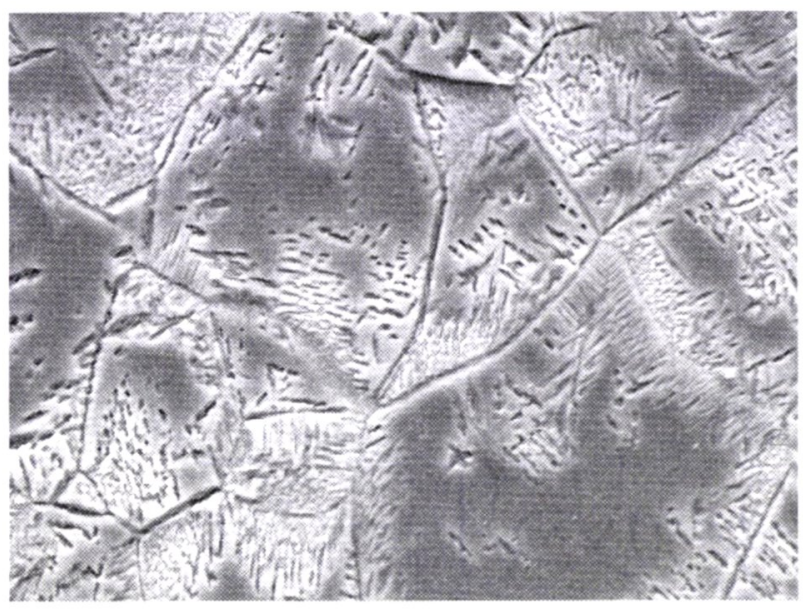

\section{R. B. Ribeiro, L.R. de O. Hein, A. Robin}

Influence of Aged Ti-15V-3Cr-3Sn-3Al Alloy Microstructure on Chemical and Electrochemical Behavior in the Kroll Reagent for Metallographic Etching

Der Einfluss des Gefüges der gealterten Legierung Ti-15V-3Cr-3Sn-3Al auf das chemische und elektrochemische Verhalten beim metallographischen Ätzen in Kroll-Reagens

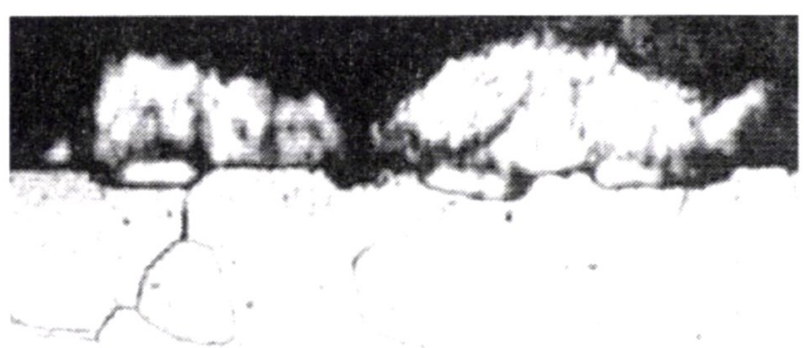

\section{H. Berndsen, F. Friedel, R. Petzold, M. Meurer, K. Benedens, K.-P. Imlau}

Oberflächenveredeltes Stahlfeinblech - eine Herausforderung an die metallografische Arbeit

Coated Steel Sheet - A Metallographical Challenge ......25

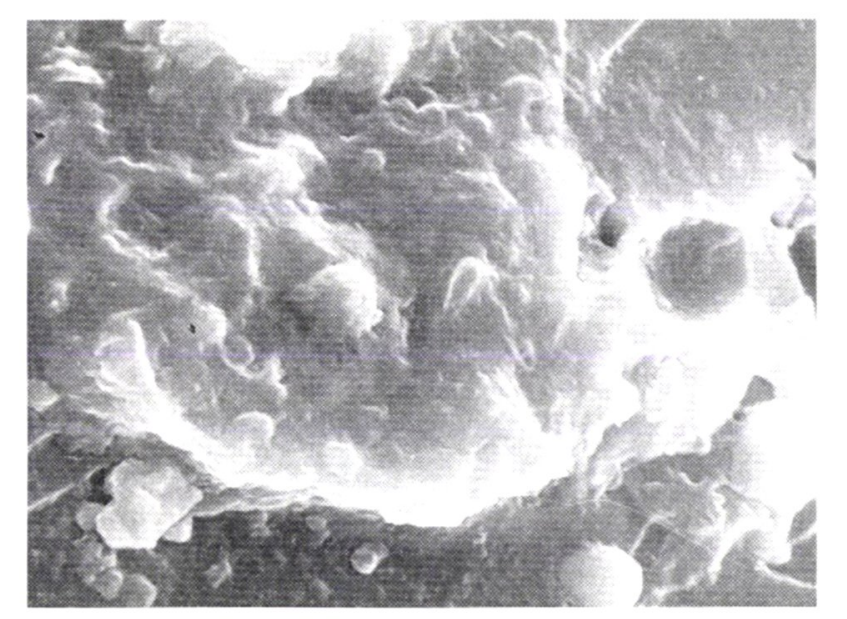

\section{Kishore, S. S. Mahajan, S. M. Kulkarni}

Fractographic Features of Dry and Water Absorbed Epoxy Based Ash Filled Particulate Composites with and without Surface Treatments for Fillers Following Short Beam Test

Fraktographische Merkmale von trockenen und gewässerten Epoxid-Teilchenverbunden mit Aschefüllung mit und ohne Oberflächenbehandlung der Füllstoffe im Biegeversuch

Editorial

Mitteilungen/Information

Heitere Metallographie/Humorous Metallography

Diskussionsforum/Topic of Discussion

Veranstaltungskalender/Meeting Diary 


\section{Praktische Metallographie} Practical Metallography

Gründer/Founders

Günter Petzow

Gerhard Reinacher †

Charlotte Wachau $\dagger$

Herausgeber/Editor

Prof. Dr. G. Petzow

Schriftleiter

Editor in charge

Prof. Dr.-Ing. F. Mücklich

Universität des Saarlandes

Lehrstuhl für Funktionswerkstoffe

Postfach 151150

D-66041 Saarbrücken

Telefon: +49/681 302-2048

Telefax: +49/681 302-4876

E-Mail: pm-editor@matsci.uni-sb.de

\section{Redaktion / Editor}

Dipl.-Ing. S. Mücklich

Zöblitzer Str. 10

D-09125 Chemnitz

Telefon: +49/371/531-5384

Telefax: +49/371/531-6179

E-Mail: silke.muecklich@mbv.tu-

chemnitz.de
Wissenschaftlicher Beirat/Editorial Board

Dr. C. Bagnall, MCS Associates Inc., Greensburg, PA (USA)

Dr. E. Bischoff, MPI für Metallforschung, Stuttgart

C. Bochert, Buehler $\mathrm{GmbH}$, Düsseldorf

Prof. Dr. H.-E. Bühler, RWTH Aachen

V. Dietl, Lette Verein Berlin

Dr. G. Elssner, MPI für Metallforschung, Stuttgart

Prof. Dr. H.E. Exner, TU Darmstadt

Prof. Dr. M. Göken, Friedrich-Alexander-Univ., Erlangen-Nürnberg

Prof. Dr. E. Hornbogen, Ruhr-Universität Bochum

Prof. Dr. F. Jeglitsch, Montanuniversität Leoben

Prof. Dr. C.-K. Kim, KUT, Korea

Dr. H.-J. Klaar, RWTH Aachen

Prof. Dr. A. KneissI, Montanuniversität Leoben

Dr. W.-U. Kopp, Daisendorf

Dr. J. Paul, Leica Vertrieb GmbH, Bensheim

Prof. Dr. M. Pohl, Ruhr-Universität Bochum

Prof. Dr. P. D. Portella, BAM, Berlin

Prof. Dr. Schmitt-Thomas, IST, München

Prof. Dr. G. Schneider, Bosch, Stuttgart

U. Täffner, MPI für Metallforschung, Stuttgart

Dr. J. Trempler, Martin-Luther-Univ. Halle-Wittenberg

Prof. Dr. H.-H. Uchida, Tokai Univ., Hiratsuka, Kanagawa

G. Vander Voort, Buehler Ltd., Illinois

Prof. Dr. B. Wielage, TU Chemnitz

Dr. H.-J. Wieland, Verein Dt. Eisenhüttenleute, Düsseldorf

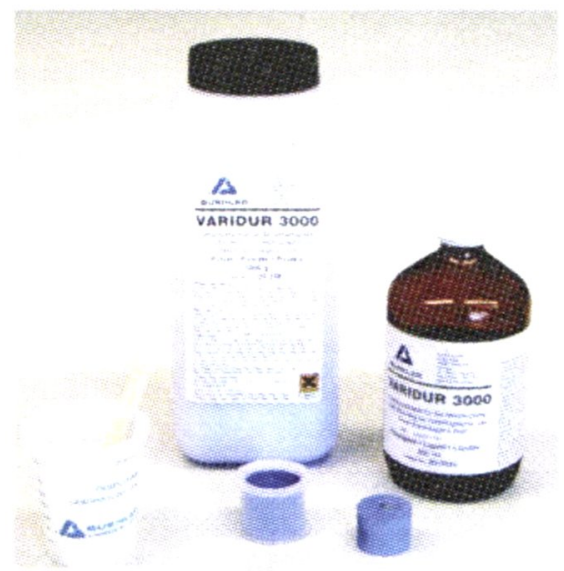

Das Jahr fängt gut an:

NEU: VARIDUR 3000 für spaltfreie Einbettungen

\section{Keine Schrumpfung, spaltfreie Schliffe Beste Randschärfe und Planheit der Probe Optimale Fließeigenschaften \\ Einfache Verarbeitung, nur zwei Komponenten}

\section{Kompetenz in Materialographie und Werkstofforüfung Bei Präparationsproblemen stehen Ihnen unsere Anwendungslabors zur Verfügung}

Buehler GmbH • In der Steele 2 • D-40599 Düsseldorf • Tel. 0211/974100 • Fax 0211/9741079 Ing. Andreas Grimas • Hauptstr. 3 • A-3012 Wolfsgraben • Tel. 02233/78610 • Fax 02233/78619 Prüfmaschinen AG • Postfach • CH-8953 Dietikon 1 • Tel. 01/7464030 • Fax 01/7464039 